\title{
Length and Clinical Effectiveness of Pulmonary Rehabilitation in Outpatients With Chronic Airway Obstruction*
}

Giuseppina Rossi, MD; Fabio Florini, RT; Micaela Romagnoli, MD;

Tommasina Bellantone, MD; Sasa Lucic, MD; Daniela Lugli, BSc; and

Enrico Clini, MD, FCCP

Study objective: To assess the clinical effectiveness of pulmonary rehabilitation (PR) after 10 or 20 consecutive sessions in outpatients with chronic airway obstruction (CAO).

Design: Observational prospective cohort trial.

Setting: Outpatient clinic of a rehabilitation center.

Patients and interventions: Twenty-five outpatients (mean age, $65 \pm 9$ years $[ \pm \mathrm{SD}] ; \mathrm{FEV}_{1}$, $64 \pm 12 \%$ predicted) admitted to a comprehensive PR program, including exercise training.

Measurements and results: The load reached on a cycloergometer (maximal achieved load [W-max]), the maximal and isoload dyspnea and leg fatigue on a Borg scale, 6 -min walk distance (6MWD), and the health-related quality of life as assessed using the St. George's Respiratory Questionnaire (SGRQ) [total and components score] have been recorded as outcome measures at baseline, after 10 sessions (T10), and after 20 sessions (T20). The predefined criteria of the clinically significant improvement were as follows: $+15 \% \mathrm{~W}$-max, $+54 \mathrm{~m}$ at $6 \mathrm{MWD},-1$ point at isoload dyspnea and leg fatigue, and $-4 \%$ at SGRQ scores. There was a mean significant difference between changes at T20 and T10 for $6 \mathrm{MWD}(-42.96 \mathrm{~m} ; 95 \%$ confidence interval [CI], -57.79 to $-28.12 \mathrm{~m} ; \mathrm{p}=\mathbf{0 . 0 0 1}$ ), total SGRQ $(4.80 ; 95 \% \mathrm{CI}, 2.29$ to $7.31 ; p=0.001)$, activity SGRQ (3.60; 95\% CI, 0.48 to $6.71 ; p=0.025)$, and symptoms SGRQ (5.96; 95\% CI, 2.72 to 9.2 ; $\mathrm{p}=0.001)$. The percentage of patients who improved was different at T20 as compared with T10 for $\mathrm{W}$-max $(68 \%$ and $48 \%$, respectively; $\mathrm{p}=0.025), 6 \mathrm{MWD}(76 \%$ and $20 \%, \mathrm{p}=0.001)$, and total SGRQ $(64 \%$ and $36 \%, p=0.008)$.

Conclusions: A 10-session course of PR provides only limited clinically significant changes of outcome measures when compared with a 20-session course in outpatients with CAO of mild-to-moderate severity.

(CHEST 2005; 127:105-109)

Key words: exercise; COPD; outcome measures; quality of life

Abbreviations: $\mathrm{CAO}=$ chronic airway obstruction; $\mathrm{CI}=$ confidence interval; GOLD $=$ Global Initiative for Obstructive Lung Disease; HRQoL = health-related quality of life; MRC = Medical Research Council; 6MWD = 6-min walk distance; $\mathrm{PR}=$ pulmonary rehabilitation; $\mathrm{SGRQ}=$ St. George's Respiratory Questionnaire; T10 = after 10 sessions; $\mathrm{T} 20=$ after 20 sessions; $\mathrm{W}$-max $=$ maximal achieved load

$\mathbf{P}$ ulmonary rehabilitation (PR) has a well-established role in the management of patients with chronic airway obstruction (CAO), particularly in those with COPD. ${ }^{1}$ Several controlled studies $^{2-4}$ have shown both short-term and long-term gains in exercise capacity and health status of these patients.

${ }^{*}$ From the Fondazione Villa Pineta and University of ModenaReggio Emilia, Division of Pneumology \& Pulmonary Rehabilitation, Pavullo (MO), Italy.

Manuscript received January 20, 2004; revision accepted August 24, 2004.

Reproduction of this article is prohibited without written permission from the American College of Chest Physicians (e-mail: permissions@chestnet.org).

Correspondence to: Enrico Clini, MD, FCCP, Ospedale e Fondazione Villa Pineta, Division of Pneumology \& Pulmonary Rehabilitation, Via Gaiato 127, 41010 Pavullo (MO), Italy; e-mail: eclini@qubisoft.it
Significant changes of outcome measures after PR have been reported in patients with $\mathrm{CAO}$ of variable severity 5,6 and in different settings. ${ }^{7}$ Nonetheless, a clinically significant improvement of these outcomes have been reported in $>50 \%$ of patients with moderate-to-severe COPD admitted to a outpatient program. ${ }^{8}$

However, the optimal length of PR in order to obtain the best clinical efficacy has still to be determined in outpatients with CAO. The initial estimates of the optimal length of outpatient PR were based on the recommendations of the American College of Sports Medicine for training in healthy individuals, which suggests that a 6 -week course is required to achieve an aerobic training effect. ${ }^{9}$

Recently, it has been shown that PR (two sessions 
per week) provides greater benefit if delivered on a 7 -week course than on a 4 -week course basis in COPD outpatients. ${ }^{10}$ In patients with COPD and mild-to-moderate degree of severity, however, there is still little to support 7 weeks (namely, 14 consecutive sessions) of training against any other reasonable length of program. Therefore, we designed an observational prospective study to compare the clinical effectiveness of PR after 10 or 20 consecutive sessions (three times per week) in outpatients with mild-to-moderate CAO. All the study procedures were conducted according to the declaration of Helsinki

\section{MATERIALS AND Methods}

\section{Patients}

The studied patients were consecutively admitted to our outpatient rehabilitation ambulatory unit. CAO was diagnosed, and the cases were classified as mild-to-moderate degree CAO; diagnosis of COPD was defined according to Global Initiative for Obstructive Lung Disease (GOLD) staging and definition, ${ }^{1}$ whereas chronic asthma was characterized by variable airflow limitation with reversible obstruction (range, 14 to 35\%) and bronchial hyperresponsiveness, ${ }^{11}$ in absence of smoking history. All the included COPD patients had history of smoking $(>20$ pack-years); four of them were current smokers.

Patients were referred directly from their general practitioner due to the worsening of symptoms. In particular, all the admitted patients complained of breathlessness (score $\geq 2$ on the 5-point Medical Research Council [MRC] scale). ${ }^{12}$ No change in the treatment with inhaled bronchodilators and/or inhaled steroids (if applied) was made in the 2 weeks preceding admission to our hospital. Patients with any severe concomitant disease (ie, severe left ventricular dysfunction, cancer), or inability or refusal to cooperate were excluded from the study.

\section{Study Protocol}

The study was an observational prospective trial. Function measures were obtained at admission (within $48 \mathrm{~h}$ ). Outcome measures were taken at baseline, after 10 sessions (T10), and after 20 session (T20) of PR. All the measurements were performed and recorded under the supervision of personnel not aware of the study purpose. Comparisons have been made among times, with each patient serving as his own control.

\section{Measurements}

Function Measures: Forced lung volumes were recorded by means of a spirometer (Masterscope; Jaeger; Hochberg, Germany). The predicted values according to Quanjer ${ }^{13}$ were used. Arterial blood to obtain $\mathrm{PaO}_{2}, \mathrm{PaCO}_{2}$, and $\mathrm{pH}$ by an automated analyzer (model 850; Chiron Diagnostics; Medfield, MA) was sampled at the radial artery, while patients in the sitting position were breathing room air for at least $1 \mathrm{~h}$. Respiratory muscle strength was assessed by measuring maximal inspiratory pressure and maximal expiratory pressure ${ }^{14}$ using a respiratory module system (Masterscope PIMAX/PEMAX Module; Jaeger). The reference values were those by Bruschi et al. ${ }^{15}$
Outcome Measures: Symptom-limited incremental exercise has been performed on a electrically braked cycloergometer (Ergometris 800-S; SensorMedics; Yorba Linda, CA) using the standard $10-\mathrm{W}$ incremental symptom-limited cycle exercise protocol. ${ }^{16}$ Mixed-expired gas data and ECG activity were monitored continuously, whereas systemic arterial BP was manually registered at each minute interval. The maximal achieved load (W-max) was recorded for analysis. At rest and at each interval, patients were asked their perceived dyspnea and leg fatigue by pointing a number or phrase on a 10 -point modified Borg scale ${ }^{17}$ set in large type on a sheet in front of them. The maximal (at baseline) and the isoload (at T10 and T20) dyspnea and leg fatigue were recorded.

The timed walk distance in meters was assessed by the 6-min walk test $(6 \mathrm{MWD})$ as recommended, ${ }^{18}$ while patients were breathing room air. Health-related quality of life (HRQoL) has been assessed by the Italian version of the St. George's Respiratory Questionnaire (SGRQ) ${ }^{19}$ : the total and each component scores (activity, symptoms, impact) of the questionnaire were measured.

\section{Rehabilitation Program}

The outpatient PR has been performed in patients admitted to a five-bed day hospital facility. After initial evaluation by chest physician and respiratory therapist, a multidisciplinary team (consisting of nurse, physical therapist, dietician, and psychologist) also assessed the patient, thus formulating an individualized rehabilitation plan. Patients performed 20 sessions (which were held up to $3 \mathrm{~h}$, three times per week) over consecutive weeks.

PR included instructions for compensatory breathing techniques, energy conservation, stress management and symptoms control, and optimization of the drug therapies. Each session included the following: (1) supervised incremental exercise on a cycloergometer; the training modality consisted of both progressively increasing load (up to 70 to $80 \%$ of the maximal load reached on the incremental test carried out at admission; a dyspnea score $<5$ determined the ability of the single patient to increase load); and time (up to 30 consecutive minutes starting from a minimum of 10 minutes $)^{20}$; (2) abdominal, and upper and lower limb muscle strength activities, lifting weights (300 to $500 \mathrm{~g}){ }^{21}$ and circling shoulders and full arms ${ }^{22}$; (3) diseaserelated education; and (4) nutritional and psychosocial counseling, when appropriate. Strategies to perform structured exercise programs at home were not implemented. During all the study period, patients were only encouraged to perform their usual daily life activities.

\section{Statistical Analysis}

Data are shown as mean values with SD and/or 95\% confidence interval (CI), frequency, and/or percentage distribution. Analysis was performed using a specific software package (SPSS 8.0 for Windows; SPSS; Chicago, IL); $\mathrm{p}<0.05$ was considered significant.

Outcomes were analyzed between times using analysis of variance for repeated measures; a post hoc test was then applied, if appropriate. The comparison of changes after 10 or 20 sessions of PR was performed using an analysis of variance model including the baseline value as the covariate; the between times differences were expressed as the mean difference for all the variables, with $95 \%$ CIs throughout.

The clinically significant improvement of the outcome measures was defined as follows: $+15 \% \mathrm{~W}$-max at the incremental cycloergometry, ${ }^{23}+54 \mathrm{~m}$ at the $6 \mathrm{MWD},{ }^{24}-1$ point at the Borg scale, ${ }^{8}$ and $-4 \%$ at each SGRQ score. ${ }^{25}$ The absolute and percentage number of patients who improved (improvers) and 
who did not (nonimprovers) were reported at any time. Analysis of difference between improvers and nonimprovers after 10 or 20 sessions was performed using the nonparametric Wilcoxon test.

\section{RESULTS}

Table 1 shows the baseline characteristics of CAO patients at hospital admission. Seven patients had chronic asthma, whereas the remaining 18 COPD patients were in GOLD stage I (mild COPD, 2 patients), GOLD stage II (moderate COPD, 11 patients), and GOLD stage III (severe COPD, 5 patients). HRQoL, as assessed by total SGRQ, showed a level of clinically significant compromise $(>10$ points) in all the patients.

The included patients were attending their first PR program, and all of them completed the 20 programmed sessions. Overall, exercise tolerance (6MWD) and capacity (W-max), and SGRQ (total, symptoms, and impact components) significantly improved at $\mathrm{T} 10(\mathrm{p}<0.01)$ and $\mathrm{T} 20(\mathrm{p}<0.01)$ as compared with baseline. Isoload dyspnea, but not isoload leg fatigue, decreased at T20 (p<0.01) [detailed data not shown].

The mean difference between changes obtained at T20 and T10 was significant for 6MWD ( $\mathrm{p}=0.001$ ), total SGRQ ( $p=0.001)$, activity SGRQ ( $p=0.025)$, and symptoms SGRQ $(p=0.001)$. Table 2 shows this analysis in detail for all the considered variables.

The number and percentage of improvers or nonimprovers after PR (see "Materials and Methods" for explanation) are shown in Table 3. The percentage of improvers at T20 was higher than at

Table 1-Baseline Demographic and Functional Data, Exercise Assessment, and Health Status*

\begin{tabular}{|c|c|}
\hline Variables & Data \\
\hline Male/female gender & $17 / 8$ \\
\hline COPD/asthma diagnosis & $18 / 7$ \\
\hline Age, yr & $65(9)$ \\
\hline Body mass index & $27(3)$ \\
\hline $\mathrm{FEV}_{1}, \%$ predicted $\dagger$ & $64(12)$ \\
\hline FVC, \% predicted $\uparrow$ & $92(15)$ \\
\hline Inspiratory capacity, $\%$ predicted $\uparrow$ & $96(16)$ \\
\hline $\mathrm{FEV}_{1} / \mathrm{FVC}, \%$ & $68(5)$ \\
\hline $\mathrm{MIP}, \mathrm{cm} \mathrm{H}_{2} \mathrm{O}$ & $67(17)$ \\
\hline $\mathrm{MEP}, \mathrm{cm} \mathrm{H}_{2} \mathrm{O}$ & $95(23)$ \\
\hline $\mathrm{W}-\max , \mathrm{W}$ & $105(29)$ \\
\hline $6 \mathrm{MWD}, \mathrm{m}$ & $448(30)$ \\
\hline Total SGRQ score & $34(14)$ \\
\hline Activity SGRQ score & $49(14)$ \\
\hline Symptoms SGRQ score & $46(18)$ \\
\hline Impact SGRQ score & $21(16)$ \\
\hline
\end{tabular}

*Data are presented as No. or mean (SD). MEP = maximal expiratory pressure; MIP = maximal inspiratory pressure.

†Postbronchodilator.
Table 2-Changes in Exercise Assessment, Symptoms, and Health Status Following 10 or 20 Rehabilitation Sessions: Data Comparisons Between T10 and T20 by Analysis of Variance With Baseline Measurements Taken as Covariates

\begin{tabular}{lrcc}
\hline \hline \multicolumn{1}{c}{ Variables } & $\begin{array}{c}\text { Mean } \\
\text { Difference }\end{array}$ & $95 \%$ CI & p Value \\
\hline W-max & -4.20 & $-8.76-0.36$ & 0.070 \\
6MWD & -42.96 & $-57.79--28.12$ & $0.001 \dagger$ \\
Dyspnea* & 0.56 & $-0.25-1.24$ & 0.105 \\
Leg fatigue* & 0.40 & $-0.47-0.55$ & 0.873 \\
Total SGRQ & 4.80 & $2.29-7.31$ & $0.001 \dagger$ \\
Activity SGRQ & 3.60 & $0.48-6.71$ & $0.025 \dagger$ \\
Symptoms SGRQ & 5.96 & $2.72-9.20$ & $0.001 \dagger$ \\
Impact SGRQ & 2.08 & $-0.89-5.04$ & 0.161 \\
\hline
\end{tabular}

*Maximal isoload values reached during cycloergometer testing. $\nmid$ Level of significance for group differences.

T10 when considering $\mathrm{W}$-max $(68 \%$ and $48 \%$, respectively; $\mathrm{p}=0.025), 6 \mathrm{MWD}(76 \%$ and $20 \%$, respectively; $\mathrm{p}=0.001$ ), and total SGRQ (64\% and $36 \%$, respectively; $\mathrm{p}=0.008$ ). Results were not influenced by diagnosis of either COPD or chronic asthma (data not shown).

\section{Discussion}

This study has shown that a 20-session course of PR including exercise training produces more significant improvements than a 10 -session course in outpatients with CAO of mild-to-moderate degree of severity. Indeed, a 10-session course of PR provides only a limited number of improvers among these patients.

Overall, this study has confirmed that comprehensive $\mathrm{PR}$ is able to improve exercise tolerance and intensity as well as symptoms and HRQoL in patients with CAO treated as outpatients., ${ }^{6,26}$ Statistically significant changes have occurred after both 10 sessions (W-max, 6MWD, and SGRQ components) and 20 sessions (W-max, 6MWD, isoload dyspnea, and SGRQ components) of PR. Nonetheless, analysis of changes between times has shown that substantial differences have arisen. Indeed, 6MWD and SGRQ (total, activity, and symptoms scores) have improved more after 20 sessions than after 10 sessions (Table 2). This fact seems rather surprising, given that the longer the program the higher are the gains potentially obtained. ${ }^{4}$

The optimal length of a PR program delivered to patients with $\mathrm{CAO}$ should not be shorter than 10 to 12 sessions. ${ }^{1}$ However, the optimal upper limit for the length of PR in these patients has not been established yet. Admission of patients with different degrees of severity and local cost-effectiveness con- 
Table 3-Patients Showing Clinical Significant Improvement After 10 or 20 Rehabilitation Sessions*

\begin{tabular}{|c|c|c|c|c|c|}
\hline \multirow[b]{2}{*}{ Variables } & \multicolumn{2}{|c|}{$\mathrm{T} 10$} & \multicolumn{2}{|c|}{$\mathrm{T} 20$} & \multirow[b]{2}{*}{$\mathrm{p}$ Value } \\
\hline & Improver & Nonimprover & Improver & Nonimprover & \\
\hline$W$-max & $12(48)$ & $13(52)$ & $17(68)$ & $8(32)$ & $0.025 \uparrow$ \\
\hline 6MWD & $5(20)$ & $20(80)$ & $19(76)$ & $6(24)$ & $0.001 \uparrow$ \\
\hline Dyspneat & $11(44)$ & $14(56)$ & $17(68)$ & $8(32)$ & 0.134 \\
\hline Leg fatiguet $+\frac{t}{+}$ & $16(64)$ & $9(36)$ & $17(68)$ & $8(32)$ & 0.739 \\
\hline Total SGRQ & $9(36)$ & $16(64)$ & $16(64)$ & $9(36)$ & $0.008 \uparrow$ \\
\hline Activity SGRQ & $12(48)$ & $13(52)$ & $15(60)$ & $10(40)$ & 0.180 \\
\hline Symptoms SGRQ & $16(64)$ & $9(36)$ & $18(72)$ & $7(28)$ & 0.414 \\
\hline Impact SGRQ & $7(28)$ & $18(72)$ & $12(48)$ & $13(52)$ & 0.061 \\
\hline
\end{tabular}

*Data are presented as No. $(\%)$.

† Level of significance for No. (\%) distribution of patients between sessions.

+ Maximal isoload values reached during cycloergometer testing.

siderations might influence the total number of allocated sessions. To the best of our knowledge, there is only one study ${ }^{10}$ trying to compare the efficacy of different lengths of PR (8 sessions vs 14 sessions). In this randomized parallel group trial ${ }^{10}$ carried out in 28 outpatients with moderate-tosevere COPD, a 7 -week course (14 sessions) of PR provides greater benefit to patients (improvement of HRQoL) than a 4 -week course (8 sessions). Unfortunately, they were not able to find any significant advantage in terms of exercise capacity by comparing the two groups.

Although the latter fact may arise from the relatively small sample size, it is rather difficult to compare results of our study with those obtained by Green et al. ${ }^{10}$ Despite a similar delivered comprehensive PR program, we have treated different patients, thus making any improvement rather comparable. A part of the treated patients in our study was in the range of mild airway obstruction. The question whether the level of bronchial obstruction per se would be "the best" measure to assess the patient's severity and to include him/her in a PR program still remains debatable. Other studies ${ }^{6,7}$ included $\mathrm{CAO}$ outpatients with a mean $\mathrm{FEV}_{1}$ similar to that of our patients. Symptoms more than the degree of airway obstruction should prompt physician to include patients into a rehabilitation program; at admission, all our patients scored $\geq 2$ on a 5 -point MRC scale. ${ }^{12}$

Notwithstanding, in the study by Green et al, ${ }^{10}$ the authors suggested that it would have been interesting to see whether results comparable to those achieved after 14 sessions could have been reached including a further 3-week period (6 sessions) of exercise training in the shorter period group. This would have determined whether the results obtained in that study represented a "lag effect," with improvements in health status developing only after a longer period of time. In this light, our study provides additional information, in that HRQoL also changed significantly after a shorter period of $\mathrm{PR}$, at least in patients with CAO of mild-to-moderate degree of severity.

Reaching the clinical threshold of improvement appears the most appropriate way to see whether any benefit can be obtained by the individual patient. ${ }^{8}$ The magnitude of change in different tools is important because that magnitude may indicate the clinical impact of the intervention. Therefore, a different way to define benefits from PR is to assess which proportion of patients has reached the clinical threshold of improvement, as regard to the considered outcome variables.

De Torres et $\mathrm{al}^{8}$ has shown that symptoms (as assessed by visual analog scale, baseline and transitional dyspnea index, and MRC scale), and HRQoL (as assessed by Chronic Respiratory Disease Questionnaire) changed to a clinically important threshold after PR in $>50 \%$ of outpatients with moderate-tosevere COPD, while other parameters dealing with exercise tolerance and intensity changed in $<50 \%$. The authors ${ }^{8}$ suggested that measurements of symptoms and HRQoL are therefore more sensitive to reflect the beneficial effect of PR.

In our study, we have found that parameters measuring exercise capacity as well as those assessing symptoms (at comparable workload) and HRQoL (here as assessed by SGRQ) may significantly change on a clinical basis in a consistent proportion of patients with CAO both after 10 sessions and 20 sessions (Table 3). However, most importantly, the percentage of patients showing such a clinically relevant improvement was different (for 6MWD, W-max, and total SGRQ) when comparing 10 sessions with 20 sessions, thus suggesting that the longer is the program the higher is the number (and percentage) of patients who may really benefit from PR, including supervised exercise.

As a matter of fact, the 20 -session course, but not the 10 -session course, provided clinical benefits in 
$>50 \%$ of patients. The percentage of improvers in exercise capacity after 20 sessions in our study was higher than that obtained by De Torres et al. ${ }^{8}$ The different diagnosis and level of impairment in the two studies might explain this result.

Our study has some limitations that need to be carefully evaluated by readers. First, the protocol is not delivered on a randomized parallel-group basis. However, when admitting outpatients with CAO to a standard PR program, we should refer all of them to a standard length, as reported in the previously published studies $^{2,6,27}$ on similar subjects. Second, the sample size is relatively small; nonetheless, the number of included patients is not much different from that of previous studies $8,10,26,27$ in this area. Additionally, all the subjects completed evaluations without any missing data. Therefore, we are confident that the obtained results might be reproducible on a larger population of CAO patients with similar characteristics.

In summary, outpatients with CAO of mild-tomoderate severity admitted to a PR program including training may largely benefit on a clinical basis if they complete a 20 -session program. Our study has shown that a 20 -session course (but not a 10 -session course) of comprehensive pulmonary rehabilitation provides clinical effectiveness in $>50 \%$ of patients, thus suggesting that a shorter length for PR would be of limited benefit. A variable cost-utility and costbenefit ratio might be therefore obtained depending on the different length of the program; further studies should be designed in order to address this important implication.

ACKNOWLEDGMENT: We thank Mrs. Gloria Penn for linguistic revision and editing of the manuscript.

\section{REFERENCES}

1 Global Initiative for Chronic Obstructive Lung Disease (GOLD). Global strategy for the diagnosis, management and prevention of chronic obstructive lung disease: NHLBI/ WHO Workshop report. Bethesda, MD: National Institutes of Health, 2001 (updated 2003); publication No. 2701. Available at: http://www.goldcopd.com/. Accessed October 14, 2004

2 Griffiths TL, Burr ML, Campbell IA, et al. Results at 1 year of outpatient multidisciplinary pulmonary rehabilitation: a randomised controlled trial. Lancet 2000; 355:362-368

3 Ries AL, Kaplan RM, Limberg TM, et al. Effects of pulmonary rehabilitation on physiologic and psychological outcomes in chronic obstructive pulmonary disease. Ann Intern Med $1995 ; 122: 823-832$

4 Lacasse Y, Brosseau L, Milne S, et al. Pulmonary rehabilitation for chronic obstructive pulmonary disease Cochrane Database Syst Rev (database online). Issue 3, 2002

5 Berry MJ, Rejeski WJ, Adair NE, et al. Exercise rehabilitation and chronic obstructive pulmonary disease stage. Am J Respir Crit Care Med 1999; 160:1248-1253

6 Foglio K, Bianchi L, Ambrosino N. Is it really useful to repeat outpatient pulmonary rehabilitation programs in patients with chronic airway obstruction? A 2-year controlled study. Chest 2001; 119:1698-1704
7 Clini E, Foglio K, Bianchi L, et al. In-hospital short-term training program for patients with chronic airway obstruction. Chest 2001; 120:1500-1505

8 De Torres JP, Pinto-Plata V, Ingenito E, et al. Power of outcome measurements to detect clinically significant changes in pulmonary rehabilitation of patients with COPD. Chest 2002; 121:1092-1098

9 American College of Sports Medicine. Guidelines for exercise testing and prescription. 4th ed. Philadelphia, PA: Lea \& Febiger, 1991

10 Green RH, Singh SJ, Williams J, et al. A randomised controlled trial of four weeks versus seven weeks of pulmonary rehabilitation in chronic obstructive pulmonary disease. Tho$\operatorname{rax} 2001 ; 56: 143-145$

11 Guidelines for the diagnosis and management of asthma: Bethesda, MD: Department of Health and Human Services, 1991; DHHS publication No. 91-3042

12 Fletcher CM. Standardised questionnaire on respiratory symptoms: a statement prepared and approved by the MRC Committee on the Aetiology of Chronic Bronchitis (MRC breathlessness score). BMJ 1960; 2:1995-1996

13 Quanjer PH. Working Party on "standardization of lung function test”. Bull Eur Physiopathol Respir 1983; 19(suppl): $7-10$

14 Black L, Hyatt R. Maximal airway pressures: normal values and relationship to age and sex. Am Rev Respir Dis 1969; 99:696-702

15 Bruschi C, Cerveri I, Zoia MC, et al. Reference values of maximal respiratory mouth pressures: a population-based study. Am Rev Respir Dis 1992; 146:790-793

16 ATS/ACCP statement on Cardiopulmonary Exercise Testing. Am J Respir Crit Care Med 2003; 167:211-277

17 Borg GAV. Psychophysical basis of perceived exertion. Med Sci Sports Exerc 1992; 14:377-381

18 American Thoracic Society. Guidelines for the six-minute walk test. Am J Respir Crit Care Med 2002; 166:111-117

19 Carone M, Bertolotti G, Anchisi F, et al. The St. George's Respiratory Questionnaire (SGRQ): Italian version. Rassegna Patol App Respir 1999; 14:31-37

20 Maltais F, LeBlanc P, Jobin J, et al. Intensity of training and physiologic adaptation in patients with chronic obstructive pulmonary disease. Am J Respir Crit Care Med 1997; 155: $555-561$

21 Simpson K, Killian K, McCartney N, et al. Randomised controlled trial of weightlifting exercise in patients with chronic airflow limitation. Thorax 1992; 47:70-75

22 Epstein SK, Celli BR, Martinez FJ, et al. Arm training reduces the $\mathrm{VO}_{2}$ and $\mathrm{VE}$ cost of unsupported arm exercise and elevation in chronic obstructive pulmonary disease. J Cardiopulmon Rehabil 1997; 17:171-177

23 Troosters T, Gosselink R, Decramer M. Exercise training in COPD: how to distinguish responders from nonresponders. J Cardiopulm Rehabil 2001; 21:10-17

24 Redelmeier DA, Bayoumi AM, Goldstein RS, et al. Interpreting small differences in functional status: the six minute walk test in chronic lung disease patients. Am J Respir Crit Care Med 1997; 155:1278-1282

25 Jones PW. Health status measurement in chronic obstructive pulmonary disease. Thorax 2001; 56:880-887

26 Foglio K, Bianchi L, Bruletti G, et al. Long-term effectiveness of pulmonary rehabilitation in patients with chronic airway obstruction. Eur Respir J 1999; 13:125-132

27 Wedzicha J, Bestall J, Garrod R, et al. Randomized controlled trial of pulmonary rehabilitation in severe chronic obstructive pulmonary disease patients, stratified with the MRC dyspnea scale. Eur Respir J 1998; 12:363-369 